\title{
Study of Higher Order Differential Mean Value Theorem for Multivariate Function
}

\author{
Wenting Ma \\ Nanchang Institute of Science \&Technology, Nanchang, Jiangxi, 330108
}

\begin{abstract}
In this paper, the Rolle theorem, Lagrange theorem and Cauchy theorem and its geometrical meanings of the multivariate functions are given. The n-th order midtheorem of the multivariate functions is also given.

Keywords: Higher Order Differential Mean Value, Multivariate Function
\end{abstract}

\section{Introduction}

The differential mean value theorem is the fundamental theorem of differential calculus and plays an important role in mathematical analysis. It is a powerful tool to study the global properties of a function in a certain interval. In this paper, we will systematically summarize the application of the mean value theorem and promote it. In this paper, we first derive the differential mean value theorem of arbitrary finite one and multivariate functions on the basis of Roll's Mean Value Theorem. Finally, the paper summarizes and illustrates several kinds of problems that can be solved by differential mean value theorem, and makes it more practical.

\section{Higher order differential mean value theorem for multivariate function}

$\mathrm{F}(\mathrm{x}), \mathrm{Ki}(\mathrm{x}), \mathrm{i}=0,1, \ldots, \mathrm{m}$ are differentiable $\mathrm{m}$ times on the convex domain $\mathrm{D} \in$ Rn. Any $a, b \in D, a \neq b$, any point column $\{x j\} m j=0 \in a b, x j=(1-t j) a+t j b, j$ 
$=0,1, \ldots, \mathrm{m}, 0=\mathrm{t} 0<\mathrm{t} 1<\mathrm{t} 2<\ldots<\mathrm{tm}=1$ is a division of $[0,1]$ And $\mathrm{Ki}(\mathrm{xj})=\mathrm{Dij}$, then there exists $\mathrm{N} \in \mathrm{ab}$

$$
\left[\sum_{i=1}^{\mathrm{n}}\left(b_{i}-a_{i}\right) D_{i}\right]^{m} f(\xi)=\sum_{i=0}^{m} f\left(x_{i}\right)\left[\sum_{i=1}^{\mathrm{n}}\left(b_{i}-a_{i}\right) D_{i}\right]^{m} \lambda(\xi)
$$

Wherein, $\mathrm{a}=(\mathrm{a} 1, \mathrm{a} 2, \cdots, \mathrm{an}), \mathrm{b}=(\mathrm{b} 1, \mathrm{~b} 2, \cdots, \mathrm{bn})$.

$\operatorname{Set} \phi(t)=f[(1-t) a+t b], \varphi(t)=\lambda[(1-t) a+t b] \mathrm{t} \in[0,1], \mathrm{i}=0,1, \cdots, \mathrm{m}$ 则 $<(\mathrm{t}), \mathrm{Ui}(\mathrm{t}), \mathrm{i}=0,1, \cdots, \mathrm{m}$ 于 $[0,1] \mathrm{m}$ minor can be noted $0=\mathrm{t} 0<\mathrm{t} 1<\mathrm{t} 2<\cdots<\mathrm{tm}=1$ is $[0$, 1] one segment, and

$$
\varphi(t)=\lambda\left[\left(1-t_{i}\right) a+t_{i} b\right]=\lambda\left(x_{i}\right)=\delta_{i j}
$$

differentiable twice on the convex domain $\mathrm{D} \in \mathrm{Rn}$, any $\mathrm{a}, \mathrm{b} \in \mathrm{D}, \mathrm{a} \neq \mathrm{b}, 1,2,0=$ $\mathrm{t} 0<\mathrm{t} 1<\mathrm{t} 2=1$ is a partition of $[0,1]$, and $\mathrm{Ki}(\mathrm{xj})=\mathrm{Dij}$, then there exists $\mathrm{N} \in \mathrm{ab}$

$$
\text { such that }(b-a) H f(\xi)(b-a)^{T}=\sum_{i=0}^{2} f\left(x_{i}\right)(b-a) H \lambda(\xi)(b-a)^{T}
$$

From the above inference, the following main conclusions can be confirmed.

Let $\mathrm{f}(\mathrm{x})$ be differentiable twice on the convex domain $\mathrm{D} \in \mathrm{Rn}$, any $\mathrm{a}, \mathrm{b}, \mathrm{c} \in \mathrm{D}, \mathrm{b}$ $\in \mathrm{ac}, \mathrm{a} \neq \mathrm{c}$, then $\mathrm{N} \in \mathrm{ac}$

$$
\begin{aligned}
& (c-a) H f(\xi)(c-a)^{T} \\
& =\frac{2}{(a-b, a-b) /(b-c, b-c)} \\
& \times\left|\begin{array}{ccc}
(b-c, a-c) & (b-c, b-c) & 0 \\
0 & (a-b, a-b) & (a-b, a-c) \\
f(a) & f(b) & f(c)
\end{array}\right|
\end{aligned}
$$

Let $\mathrm{a}=\mathrm{x} 0, \mathrm{~b}=\mathrm{x} 1, \mathrm{c}=\mathrm{x} 2$, from the inference 2.2 , there $\mathrm{N} \in \mathrm{ac}$, so

$$
\text { that } \begin{aligned}
(c-a) H f(\xi)(c-a)^{T}=\sum_{i=0}^{2} f\left(x_{i}\right)(b-a) H \lambda(\xi)(c-a)^{T} \\
\lambda_{0}(x)=\frac{(a-b, x-b)(a-c, x-c)}{(a-b, a-b)(a-c, a-c)} \\
\lambda_{1}(x)=\frac{(b-a, x-a)(b-c, x-c)}{(b-a, b-a)(b-c, b-c)} \\
\lambda_{2}(x)=\frac{(c-a, x-a)(c-b, x-b)}{(c-a, c-a)(c-b, c-b)}
\end{aligned}
$$

Then 


$$
\begin{aligned}
& (c-a) H f(\xi)(c-a)^{T} \\
& =2 f(a) \frac{(a-b, x-b)(a-c, x-c)}{(a-b, a-b)(a-c, a-c)} \\
& +2 f(b) \frac{(b-a, x-a)(b-c, x-c)}{(b-a, b-a)(b-c, b-c)} \\
& +2 f(c) \frac{(c-a, x-a)(c-b, x-b)}{(c-a, c-a)(c-b, c-b)} \\
& =\frac{2}{(a-b, a-b)(b-c, b-c)} \\
& \times\left|\begin{array}{ccc}
(b-c, a-c) & (b-c, b-c) & 0 \\
0 & (a-b, a-b) & (a-b, a-c) \\
f(a) & f(b) & f(c)
\end{array}\right|
\end{aligned}
$$

\section{The Application Research of Mean Value Theorem of Differential}

Here, we first show the difference between $\theta$ and $\xi$, where $\xi$ is a constant in the domain $(a, b)$, and $\theta$ is the extent of the location of $\xi$ in the domain. The range is $(0,1)$, is the median value, is the median value of $\xi$, the specific relationship is $\xi$ $=\mathrm{a}+$ (ba) $\theta$, it is called the median value. In the title of the requirements are the limit of $\theta$, then, there are two ways to find this limit:

1 , when $\theta$ can be directly expressed by other variables, when the problem is a set of purely limit the subject.

2 , when $\theta$ can not be directly expressed by the definition of derivatives, multiplication and division in the patchwork multiplication and elimination of offset, $\theta$ will appear in the molecule. When constructing derivative definitions, we need to use the Newtonian Leibniz formula in conjunction with Lagrange's theorem or Taylor's Mean Value Theorem (sometimes based on the first elimination of a Taylor effect).

The following specific description. When there is one or two integral upper bound functions on one side of the problem to be proof equation, when there is no, then we must first remove the integral symbol. At this point we should pay attention to two issues, if there is more than one integral upper limit function, the first method to be replaced by the two integral type of unity, because the two integral will appear two median, and the two median There is no relationship, so at this time points to unity. Then, we know that there are two ways to the integration number, one Newton Leibniz formula, one is the integral mean value theorem. The mean value theorem states explicitly that the value lies above the 
closed interval, which means that the median may be obtained at the endpoint, but we look back at $\theta$, which is in the $(0,1)$ open interval, Thus, $\xi$ lies in the $(a, b)$ open interval, which also corresponds to the content of the mean value of the differential mean value theorem in the open interval. Thus, remove the integral symbol here is not to use the integral mean value theorem, To use the Newton Leibniz formula. Using the Newton Leibniz formula, there will be F (x) -F (a) situation, this logical use of Lagrange theorem theorem $\theta$ appears naturally, finishing can be proved. The bounds are then determined by combining derivative definitions. When the condition is n-order continuous derivative, where a certain derivative can not be zero, and a $\mathrm{f}(\mathrm{x}+\mathrm{h})$ function, we know that this time we must use the Taylor theorem with derivative definition solution. Taylor formula here, unlike other types of topics to be simultaneously launched two times and then eliminate the yuan, where only once, is to give the equation to do elimination, the elimination formula, if it is seen that the derivative definition, structural derivatives Definition of $\theta$, then Taylor residual is removed by the division. If there are trivial items, then the elimination of Taylor do expand, and consumer elimination, the definition of derivative structure to find $\theta$, Taylor residual is subtracted at this time subtracted. There is a small mark, that is, if the subject clearly shows that a number is not zero, then it is likely to become a divisor. This question type is completed.

In fact, the auxiliary function is to be a function of the original function, with the knowledge of differential equations to find a proof function of the original function is also possible, but the content of the problem is only enough to restore the $\ln$ function, there is no need to introduce differential equations . Find the original function, back to type two on it. In addition, if the problem people want to engage in complex, may split the two-tier function and the form of or poor form, when we open to the subject of the derivative of a third-order second order (321 or 210) Three of the first order difference between the situation, we want to merge them. In the combined form to find the source function. Now back to type two, with the function, with a closed interval on the open interval can lead to the conditions, but also need to find two equal values of the function point. The point-finding method discussed in Type II is still used. Since the derivative of the original function is a relatively complex function, the method of the problem is also relatively more. The equivalent point here is of course the source function of the equivalent point, the title may be directly to the source function of the equivalent point, the source function may also be a derivative of the child, continue to bring the source and then find the equivalent point. The following methods I have seen summed up.

1, the given function points directly into the source function2, first with Rollel theorem to determine a derivative of the zero point, and then talk about this point into the source function.

3 , the condition for the definite integral form, the integral mean value theorem can be used to find a point, or turn it into a variable upper limit function, then Newton Leibniz formula to get the same point. Finally, there are functional equations, there are two equivalent points, can be used Rolle's theorem. This question type is completed. 
Still and the type of two, three, four of the general idea has been here, more than four types of questions a step, that is, the problem will be clear to deliberately disrupt the form, resulting in a function value, the value of the variable, the median scattered We know that this is still the disciples of Roll's theorem, first sorting out the formula, into a zero equation, and then look at the equation (1), then we can see, Who is the source side of the function, and the type of questions about the same four. Note that this form of the original function containing the parameters is accumulated. This question type is completed.

(A), $f(b)$, the median and the letter points can be separated from both sides of the equation (the process rate of change must have a value to meet the state rate of change )

The above two, three, four, five types of problems are derived from the conditions and conclusions Luoer theorem evolved, respectively, the most primitive type, hidden source function type ln method, the implicit source function formula type, hidden parameter source Function formula type, we will begin to introduce the following by the Lagrange constant force evolution of the problem type.

See the title six I am very happy, because the title is good, you look at the five questions and the type of problem is not like six, the difference is only the value of the letters can be separated. This is precisely the one leading us from the Rolle theorem trace to Lagrange Lagrange force of a bridge ah. We can go back to the previous article to see the difference between the two fixed-force where. I would simply say that theorem of Rohr theorem states that the values of the two functions of the closed derivative are equal and that there must be a value in the open interval such that this value corresponds to a derivative function of zero. Lagrangian force conditions $\mathrm{Bi}$ Lian enlighten, concluded that there must be some point within the open process is equal to the rate of change of state change rate. Note that the Lagrangian theorem compared to Roll's theorem, the conditions are less, more conclusions. And less and more are related to $\mathrm{f}$ (a), f (b) related things. The essential difference between the two kinds of questions is the essential difference between them. When we find that there is no way to separate the function value and the median value, they are a whole and are obtained after a whole derivation. Under the only zero, the overall derivative is equal to zero, which of course is Rolle's theorem. And if you can separate, which means that the corresponding value of that part of the function of the source function when the derivative may not be zero, That is to say the remaining part is Lagrange is the conclusion of the extra part, So this is the Lagrangian type, This is the difference ah. We can also look at the characteristics of this type if not separate, and other Roll theorem exercises, we can find information about the function of the value of the points, so as to find the equivalent point application of Rolle's theorem, and if you can split, then There is no information on the value of the function point, because the Lagrange force itself does not require the equivalent conditions. We know that the Cauchy mean value theorem is a form of parametric equation of Lagrange mean value theorem, that is, $y=f(x)$ and $x=g(t) y=h(t)$ We have to emphasize that the function in the denominator position is not equal to zero because of the division property. Questions in the form of six may also be 
Cauchy, but the same nature. The last is to find the source function. Note the derivative form of the multiplication and division equations. This question type is completed.

\section{References}

[1] Chen Ping, Wan Xianglan. Differential Mean Value Theorem and Its Application Example . Examiner Week, 5(2), pp. 22-23, 2016

Application of differential mean value theorem. Science and Technology of Vision, 22(2), pp. 20-25, 2015

[2] Xiang Changfu. The Teaching Research of Differential Mean Value Theorem .Qujing Normal University, 3(2), pp. 20-25, 2014.

[3] Wang Yuhua, Li Jian-hua. Fuzzy Confusion Problems in Differential Mean Value Theorem , 8 (11), pp. 123-125, 2013.

[4] Zhang Lili, Qi Yuan. Discussion on Inverse of Mean Value Theorem of Differential . Journal of Longdong University, 5(2), pp. 18-23, 2003

[5] Wang Shenqiu, Fan Zhenbin. Asymptotic analysis of the mean value theorem for differential mean value theorem. Changshu Electric Appl. Journal of University of Science and Technology, 2(2), pp. 26-35, 2012 